\title{
Guidance Law and Neural Control for Hypersonic Missile to Track Targets
}

\author{
Wenxing Fu, Binbin Yan, Xiaofei Chang, and Jie Yan \\ School of Astronautics, Northwestern Polytechnical University, Xian 710072, China \\ Correspondence should be addressed to Wenxing Fu; wenxingfu@nwpu.edu.cn \\ Received 17 October 2015; Revised 22 December 2015; Accepted 14 January 2016 \\ Academic Editor: Filippo Cacace
}

Copyright (C) 2016 Wenxing Fu et al. This is an open access article distributed under the Creative Commons Attribution License, which permits unrestricted use, distribution, and reproduction in any medium, provided the original work is properly cited.

\begin{abstract}
Hypersonic technology plays an important role in prompt global strike. Because the flight dynamics of a hypersonic vehicle is nonlinear, uncertain, and highly coupled, the controller design is challenging, especially to design its guidance and control law during the attack of a maneuvering target. In this paper, the sliding mode control (SMC) method is used to develop the guidance law from which the desired flight path angle is derived. With the desired information as control command, the adaptive neural control in discrete time is investigated ingeniously for the longitudinal dynamics of the hypersonic missile. The proposed guidance and control laws are validated by simulation of a hypersonic missile against a maneuvering target. It is demonstrated that the scheme has good robustness and high accuracy to attack a maneuvering target in the presence of external disturbance and missile model uncertainty.
\end{abstract}

\section{Introduction}

The hypersonic missile can propel the development of the prompt global strike. Controller design is crucial in making it feasible. To control the hypersonic vehicle whose flight dynamics is nonlinear, uncertain, and coupled, many researches were carried out. In [1], the development of flight dynamics and controller design is comprehensively reviewed.

The SMC is used to control a generic hypersonic vehicle in [2]. The dynamics of the hypersonic vehicle is decoupled to velocity and attitude subsystems [3] that have been transformed into the linearly parameterized form. Considering the parametric model uncertainty and input saturations, the dynamic inverse control is presented via the backstepping design in which the dynamic surface control is used. Furthermore, the dead-zone input is considered in [4] with Nussbaum gain based controller design. In [5], the aerothermoelastic effect is taken into consideration in the hypersonic vehicle model, and a robust controller is developed to achieve global exponential tracking of the reference model. Fixedorder $\mu$ controllers are designed to constrain the controller's dimensions with the propulsion system perturbations and aeroelastic fuselage bending considered as uncertainties in [6]. A coupled linear parameter varying (LPV) and flatness design is proposed to solve the hypersonic guidance problem in [7]. In [8,9], robust control using the genetic algorithm to search a design coefficient space is proposed.

In these literatures, the control outputs are flight velocity and altitude which are driven to track the reference trajectory that increases from zero to constant value after a transient phase. When the hypersonic missile is used to attack targets, the dynamics will change greatly in the endgame phase due to the angle and angle rate changes. More problems exist in the control of the hypersonic missile in attack scenarios, and researchers began to pay more attention to them. The control of the hypersonic missile attacking a ground stationary target with a different vector-output and time-varying commanded output trajectories is using nonlinear control [10] and adaptive control [11] separately.

In this paper, the guidance and control law of a hypersonic missile attacking a maneuvering target instead of a ground stationary target are discussed. It is a more challenging task owing to the uncertainties of target maneuvers. To solve this problem, the SMC guidance law and the backstepping control law are designed to guarantee the robustness. The SMC method is one of the robust control design methods which is robust to parameter perturbations and 
external disturbances $[12,13]$. Reference [14] proposed the switched bias proportional navigation guidance law which is derived by using the sliding mode control theory based on proportional navigation [15]. In [16], an adaptive sliding mode guidance law designed for intercepting a maneuvering target is proposed. It is robust in the presence of disturbances and parameter perturbations and is able to eliminate chattering. With target acceleration considered as bounded uncertainty, a sliding mode guidance law is derived based on the nonlinear planar engagement kinematics in [17]. In [18], the sliding mode control method with the zero-effort miss distance considered as the sliding surface is derived. In [19], a novel smooth second-order sliding mode (SSOSM) control was applied to guidance law design. Compared with augmented proportional navigation guidance law, the SSOSM can achieve better miss distance performance.

Back-stepping design [20-22] is a powerful tool to design the controller for a nonlinear system in parameter strictfeedback form. Properties of strong stability and tracking are built into the nonlinear system in a number of steps, which are never higher than the system order. In [23], the sequential loop closure controller design decomposing equations into functional subsystems is adopted. Moreover, intelligent control receives increasing attention since the neural networks [24-28] or fuzzy logic system $[29,30]$ can learn the dynamics efficiently. In [31], the global design with neural approximation is proposed for both indirect and direct control of hypersonic dynamics.

With the development of hardware, the research on discrete-time control has received a considerable attention [32-35]. As pointed out in [36], the use of digital computers and samplers in control circuitry has made the use of discrete-time system representation more justifiable than continuous-time system representation. For the control of a flight vehicle, a controller using continuous-time system is usually implemented by a digital computer with a certain sampling interval $[37,38]$. As for the modeling, the discrete strict-feedback form of HFV model is obtained with the Euler approximation [39]. Focusing on the uncertainty estimation, the adaptive back-stepping discrete controller is investigated for hypersonic flight [40].

In this paper, we first design the sliding mode guidance law from which the desired flight path angle is derived. Then, we design the controller with the back-stepping scheme recursively. We take into consideration the nominal nonlinearity for feedback design and the neural network (NN) is used to approximate the system uncertainty. In order to avoid the circular construction of control inputs, the upper bound is taken instead of the nominal value for coefficient design. The idea of the guidance and control design is shown in Figure 2. This paper is organized as follows. Section 2 briefly presents the longitudinal dynamics of a generic HFV. Section 3 presents the guidance law design via sliding mode control. The system transformation of prediction model is given in Section 4. Section 5 presents the adaptive neural controller design and the stability analysis for the subsystems, respectively. The target interception simulation is included in Section 6. Section 7 presents several comments and final remarks.

\section{Hypersonic Vehicle Modeling}

This model comprises five state variables $X_{h}=[V, h, \alpha, \gamma, q]^{T}$ and two control inputs $U_{c}=\left[\delta_{e}, \beta\right]^{T}$, where $V$ is the velocity, $\gamma$ is the flight path angle, $h$ is the altitude, $\alpha$ is the angle of attack, $q$ is the pitch rate, $\delta_{e}$ is elevator deflection, and $\beta$ is the throttle setting:

$$
\begin{aligned}
& \dot{V}=\frac{T \cos \alpha-D}{m}-\frac{\mu \sin \gamma}{r^{2}}, \\
& \dot{h}=V \sin \gamma, \\
& \dot{\gamma}=\frac{L+T \sin \alpha}{m V}-\frac{\left(\mu-V^{2} r\right) \cos \gamma}{V r^{2}}, \\
& \dot{\alpha}=q-\dot{\gamma}, \\
& \dot{q}=\frac{M_{y y}}{I_{y y}} .
\end{aligned}
$$

The definitions of the dynamics of hypersonic flight vehicle (HFV) in [41] are given as follows: $r=h+R_{E}, \bar{q}=(1 / 2) \rho V^{2}$, $L=\bar{q} S C_{L}, D=\bar{q} S C_{D}, T=\bar{q} S C_{T}, M_{y y}=\bar{q} S \bar{c}\left[C_{M}(\alpha)+\right.$ $\left.C_{M}\left(\delta_{e}\right)+C_{M}(q)\right], C_{L}=0.6203 \alpha, C_{D}=0.6450 \alpha^{2}+$ $0.0043378 \alpha+0.003772, C_{M}(\alpha)=-0.035 \alpha^{2}+0.036617 \alpha+$ $5.3261 \times 10^{-6}$, and $C_{M}(q)=(\bar{c} / 2 V) q\left(-6.796 \alpha^{2}+0.3015 \alpha-\right.$ 0.2289 ).

The control input is defined as

$$
\begin{aligned}
C_{T} & = \begin{cases}0.02576 \beta & \text { if } \beta<1 \\
0.0224+0.00336 \beta & \text { otherwise, }\end{cases} \\
C_{M}\left(\delta_{e}\right) & =0.0292(\delta e-\alpha),
\end{aligned}
$$

where $\rho$ denotes the air density, $S$ is the reference area, $\bar{c}$ is the reference length, and $R_{E}$ is the radius of the Earth. $C_{x}$, $x=L, D, T, M$, are the force and moment coefficients.

\section{Guidance Law Design}

The geometry of an idealized interception in which the missile and the target are closing on each other at constant speed is shown in Figure 1, where $M$ is hypersonic missile, $T$ is target, $V_{M}$ and $\gamma_{M}$ are the speed and path angle of the hypersonic missile, $V_{T}$ and $\gamma_{T}$ are the speed and path angle of the target, $r$ is the distance between the hypersonic missile and the target, and $\lambda$ is the line of sight (LOS). Then, the LOS rate can be represented as

$$
\dot{\lambda}=\frac{1}{r}\left(V_{M} \sin \left(\lambda-\gamma_{M}\right)-V_{T} \sin \left(\lambda-\gamma_{T}\right)\right) .
$$

The crucial part of the sliding mode controller design is the sliding surface design. To attain a collision triangle, the angle rate command of the missile is usually chosen to be proportional to the LOS rate when the speeds of the interceptor and the target are constants. So the switching function can be chosen as

$$
s=r \dot{\lambda} .
$$




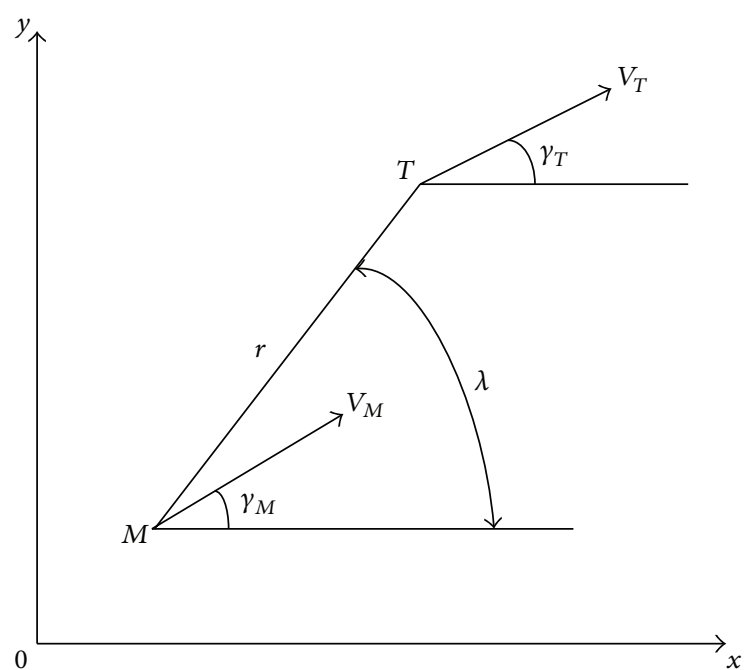

FIGURE 1: Interception geometry.

As mentioned before, the missile and the target are closing on each other at constant speed. Then, the exponential approximation law can be used to achieve satisfactory dynamical performance. The adaptive approximation law can be designed as

$$
\dot{s}=-k s-\varepsilon \operatorname{sgn} s,
$$

where $k$ and $\varepsilon$ are positive constants and $\operatorname{sgn}()$ is defined as

$$
\operatorname{sgn} s= \begin{cases}1, & s>0 \\ 0, & s=0 \\ -1, & s<0 .\end{cases}
$$

To prevent the inertia of a constant approximation law from making the LOS angular rate diverge, the adaptive approximation law is designed as

$$
\dot{s}=-\frac{k|\dot{r}|}{r} s-\varepsilon \operatorname{sgn} s .
$$

Equation (11) means that the sliding mode approximation speed is adjusted by the target-to-missile ranger $r$. When $r$ is close to zero, the speed increases rapidly to prevent $\dot{\lambda}$ from diverging. To mitigate chattering, a continuous function $\dot{\lambda} /(|\dot{\lambda}+\delta|)$ can be a substitute for the variable structure item $\operatorname{sgn} \dot{\lambda}$, where $\delta$ is a small positive real number. Thus, the sliding mode guidance law is written as

$$
n_{y}=(k+1)\left|\dot{V}_{R}\right| \dot{\lambda}+\frac{\varepsilon \dot{\lambda}}{(|\dot{\lambda}|+\delta)} .
$$

Substitute (12) into dynamic equation

$$
n_{y}=\frac{V}{g} \dot{\gamma}
$$

By integrating $\dot{\gamma}$, we can obtain the desired path angle $\gamma_{d}$.

\section{System Transformation}

Equations (1)-(5) are applicable when the velocity is mainly related to throttle setting and the rate of altitude change is controlled by the elevator deflection. So the dynamics of the HFV can be decoupled into two functional subsystems. With the tracking references $V_{d}$ and $h_{d}$, we design the velocity and altitude controller separately in Section 5 . The schematic diagram is depicted in Figure 2.

\subsection{Strict-Feedback Formulation}

Assumption 1. Since $\gamma$ is small, we can take $\sin \gamma \approx \gamma$ in (2) for simplification. The thrust term $T \sin \alpha$ in (3) can be neglected because it is generally much smaller than $L$.

Assumption 2. The velocity can be considered as constant during the hypersonic missile attacking the target.

4.1.1. Velocity Subsystem. The velocity subsystem (1) can be rewritten into the following form:

$$
\begin{aligned}
\dot{V} & =f_{V}+g_{V} u_{V}, \\
u_{V} & =\beta, \\
y_{V} & =V,
\end{aligned}
$$

where $f_{V}=-\left(D / m+\mu \sin \gamma / r^{2}\right)+\bar{q} S \times 0.0224 \cos \alpha / m$ and $g_{V}=\bar{q} S \times 0.00336 \cos \alpha / m$ if $\beta>1$. Otherwise, $f_{V}=-(D / m+$ $\left.\mu \sin \gamma / r^{2}\right)$ and $g_{V}=\bar{q} S \times 0.02576 \cos \alpha / m$.

4.1.2. Altitude Subsystem. The altitude tracking error is defined as $\widetilde{h}=h-h_{d}$ and the flight path command is chosen as

$$
\gamma_{d}=\arcsin \left[\frac{-k_{h}\left(h-h_{d}\right)-k_{I} \int\left(h-h_{d}\right) d t+\dot{h}_{d}}{V}\right] .
$$

If $k_{h}>0$ and $k_{I}>0$ are chosen and the flight path angle is controlled to follow $\gamma_{d}$, the altitude tracking error is regulated to zero exponentially [42].

Define $\mathbf{X}_{A}=\left[x_{1}, x_{2}, x_{3}\right]^{T}, x_{1}=\gamma, x_{2}=\theta_{p}$, and $x_{3}=q$, where $\theta_{p}=\alpha+\gamma$. Then, the strict-feedback form equations of the attitude subsystem (3)-(5) are written as

$$
\begin{aligned}
& \dot{x}_{1}=f_{1}\left(x_{1}\right)+g_{1}\left(x_{1}\right) x_{2}, \\
& \dot{x}_{2}=f_{2}\left(x_{1}, x_{2}\right)+g_{2}\left(x_{1}, x_{2}\right) x_{3}, \\
& \dot{x}_{3}=f_{3}\left(x_{1}, x_{2}, x_{3}\right)+g_{3}\left(x_{1}, x_{2}, x_{3}\right) u_{A}, \\
& u_{A}=\delta_{e}, \\
& y=x_{1},
\end{aligned}
$$

where $f_{1}=-\left(\mu-V^{2} r\right) \cos \gamma /\left(V r^{2}\right)-0.6203 \bar{q} S \gamma /(m V), g_{1}=$ $0.6203 \bar{q} S /(m V), f_{2}=0, g_{2}=1, f_{3}=\bar{q} S \bar{c}\left[C_{M}(\alpha)+C_{M}(q)-\right.$ $0.0292 \alpha] / I_{y y}$, and $g_{3}=0.0292 \bar{q} S \bar{c} / I_{y y}$. 


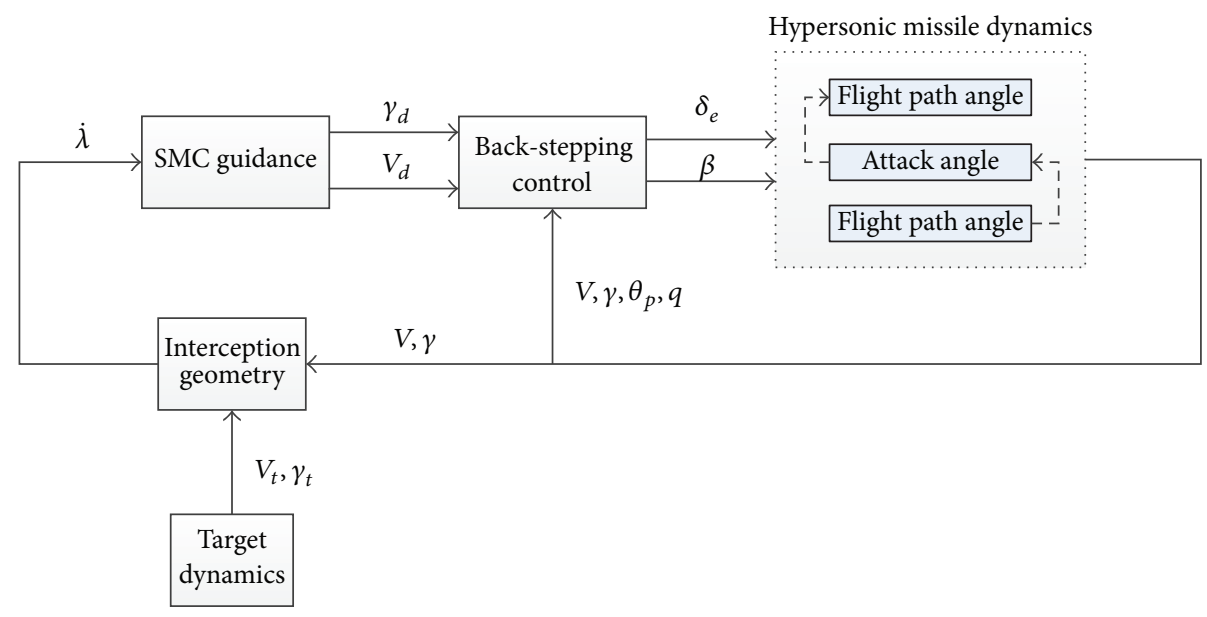

FIgURE 2: The control schematic diagram.

Assumption 3. $f_{i}$ and $g_{i}$ are unknown smooth functions. There exist constants $\bar{g}_{i}$ and $\underline{g}_{i}$ such that $\bar{g}_{i} \geq g_{i} \geq \underline{g}_{i}>0$, $i=1,3, V$.

4.2. Discrete-Time Model. With the Euler expansion for the sampling period $T_{s}$, systems (14) and (16) can be approximated as

$$
\begin{aligned}
& V(k+1)=V(k)+T_{s}\left[f_{v}(k)+g_{v}(k) u_{v}(k)\right], \\
& x_{1}(k+1)=x_{1}(k)+T_{s}\left[f_{1}(k)+g_{1}(k) x_{2}(k)\right], \\
& x_{2}(k+1)=x_{2}(k)+T_{s}\left[f_{2}(k)+g_{2}(k) x_{3}(k)\right], \\
& x_{3}(k+1)=x_{3}(k)+T_{s}\left[f_{3}(k)+g_{3}(k) u_{A}(k)\right] .
\end{aligned}
$$

\section{Discrete-Time Controller Design}

(1) Define $z_{1}(k)=x_{1}(k)-x_{1 d}(k)$, where $x_{1 d}(k)=\gamma_{d}(k)$ is derived from (15). Then,

$$
\begin{aligned}
z_{1}(k+1)= & x_{1}(k)+T_{s}\left[f_{1}(k)+g_{1}(k) x_{2}(k)\right] \\
& -x_{1 d}(k+1) .
\end{aligned}
$$

The desired control input is

$$
\begin{aligned}
& x_{2 d}^{*}(k)=\frac{1}{T_{s} g_{1}(k)}\left(c_{1} z_{1}(k)-T_{s} f_{1}(k)-x_{1}(k)\right. \\
& \left.\quad+x_{1 d}(k+1)\right) .
\end{aligned}
$$

However, it can be observed that functions $f_{1}(k)$ and $x_{1 d}(k+$ $1)$ are unknown. Thus, the uncertainty is defined and approximated by $\mathrm{NN}$ :

$$
\begin{aligned}
U_{1}(k) & =-T_{s} f_{1}(k)-x_{1}(k)+x_{1 d}(k+1) \\
& =\omega_{1}^{* T} \theta_{1}\left(X_{1}(k)\right)+\varepsilon_{1}\left(X_{1}(k)\right),
\end{aligned}
$$

where $\omega_{1}^{*}$ is the optimal $\mathrm{NN}$ weight vector to approximate $U_{1}(k)$ and $X_{1}(k)=\left[V(k), x_{1}(k), x_{2}(k), x_{3}(k), x_{d}(k)\right]^{T}$. Denoting $\theta_{1}(k)=\theta_{1}\left(X_{1}(k)\right), \varepsilon_{1}(k)=\varepsilon_{1}\left(X_{1}(k)\right)$ is the $\mathrm{NN}$ constriction error with the upper bound $\varepsilon_{1 M}$.
The virtual controller $x_{2 d}(k)$ is designed with the following form:

$$
x_{2 d}(k)=\frac{1}{T_{s} g_{1}(k)}\left(c_{1} z_{1}(k)+\widehat{\omega}_{1}^{T} \theta_{1}\left(X_{1}(k)\right)\right),
$$

where $\widehat{\omega}_{1}$ is the estimation of $\omega_{1}^{*}$ and $c_{1}$ is the design constant. know

Define $z_{2}(k)=x_{2}(k)-x_{2 d}(k)$. From (19) and (22), we

$$
\begin{aligned}
z_{1}(k+1)= & c_{1} z_{1}(k)+T_{s} g_{1}(k) z_{2}(k)+\widetilde{\omega}_{1}^{T}(k) \theta_{1}(k) \\
& -\varepsilon_{1}(k),
\end{aligned}
$$

where $\widetilde{\omega}_{1}=\widehat{\omega}_{1}-\omega_{1}^{*}$.

The NN updating law is proposed as

$$
\widehat{\omega}_{1}(k+1)=\widehat{\omega}_{1}(k)-\lambda_{1} z_{1}(k+1) \theta_{1}(k)-\delta_{1} \widehat{\omega}_{1}(k) .
$$

Define the Lyapunov function candidate:

$$
L_{1}(k)=z_{1}^{2}(k)+\frac{\widetilde{\omega}_{1}^{T}(k) \widetilde{\omega}_{1}(k)}{\lambda_{1}} .
$$

The first difference is calculated as

$$
\begin{aligned}
\Delta L_{1}(k)= & L_{1}(k+1)-L_{1}(k) \\
= & z_{1}^{2}(k+1)-z_{1}^{2}(k) \\
& +\frac{\widetilde{\omega}_{1}^{T}(k+1) \widetilde{\omega}_{1}(k+1)-\widetilde{\omega}_{1}^{T}(k) \widetilde{\omega}_{1}(k) .}{\lambda_{1}} .
\end{aligned}
$$

From (24), it is obvious that

$$
\widetilde{\omega}_{1}(k+1)=\widetilde{\omega}_{1}(k)-\lambda_{1} z_{1}(k+1) \theta_{1}(k)-\delta_{1} \widehat{\omega}_{1}(k) .
$$

From (23), we have

$$
\begin{aligned}
\widetilde{\omega}_{1}^{T}(k) & \theta_{1}(k) z_{1}(k+1) \\
= & z_{1}^{2}(k+1)-T_{s} g_{1}(k) z_{1}(k+1) z_{2}(k) \\
& \quad-c_{1} z_{1}(k) z_{1}(k+1)+\varepsilon_{1}(k) z_{1}(k+1) .
\end{aligned}
$$


It is known that $\left\|\theta_{1}(k)\right\|^{2} \leq N_{1}$, where $N_{1}$ is the number of NN nodes. Now, we have

$$
\begin{aligned}
\Delta L_{1}(k)= & L_{1}(k+1)-L_{1}(k) \\
\leq & z_{1}^{2}(k+1)-z_{1}^{2}(k)+\lambda_{1} N_{1} z_{1}^{2}(k+1) \\
& +\frac{\delta_{1}^{2}}{\lambda_{1}}\left\|\widehat{\omega}_{1}(k)\right\|^{2}-2 z_{1}^{2}(k+1) \\
& +2 T_{s} g_{1}(k) z_{1}(k+1) z_{2}(k) \\
& +2 c_{1} z_{1}(k) z_{1}(k+1)-2 \varepsilon_{1}(k) z_{1}(k+1) \\
& -2 \frac{\delta_{1}}{\lambda_{1}} \widetilde{\omega}_{1}^{T}(k) \widehat{\omega}_{1}(k) \\
& +2 \delta_{1} \widehat{\omega}_{1}^{T}(k) \theta_{1}(k) z_{1}(k+1) .
\end{aligned}
$$

The following inequalities are obtained:

$$
\begin{aligned}
& 2 \varepsilon_{1}(k) z_{1}(k+1) \leq \rho_{11} \varepsilon_{1 M}^{2}+\frac{1}{\rho_{11}} z_{1}^{2}(k+1), \\
& 2 z_{1}(k+1) z_{1}(k) \leq \rho_{12} z_{1}^{2}(k+1)+\frac{1}{\rho_{12}} z_{1}^{2}(k), \\
& 2 z_{2}(k) z_{1}(k+1) \leq \rho_{13} z_{2}^{2}(k)+\frac{1}{\rho_{13}} z_{1}^{2}(k+1), \\
& 2 \widetilde{\omega}_{1}^{T}(k) \widehat{\omega}_{1}(k)=\widetilde{\omega}_{1}^{T}(k) \widetilde{\omega}_{1}(k)+\left\|\widehat{\omega}_{1}(k)\right\|^{2}-\left\|\omega_{1}^{*}\right\|^{2}, \\
& 2 \widehat{\omega}_{1}^{T}(k) \theta_{1}(k) z_{1}(k+1) \\
& \quad \leq \rho_{14}\left\|\widehat{\omega}_{1}(k)\right\|^{2}+\frac{1}{\rho_{14}} N_{1} z_{1}^{2}(k+1),
\end{aligned}
$$

where $\rho_{1, i}, \quad i=1,2,3,4$, are positive scalars.

The first difference of $L_{1}(k)$ is obtained as

$$
\begin{aligned}
\Delta L_{1}(k) \leq & z_{1}^{2}(k+1)-z_{1}^{2}(k)+\lambda_{1} N_{1} z_{1}^{2}(k+1) \\
& +\frac{\delta_{1}^{2}}{\lambda_{1}}\left\|\widehat{\omega}_{1}(k)\right\|^{2}-2 z_{1}^{2}(k+1) \\
& +\rho_{13} T_{s} \bar{g}_{1} z_{2}^{2}(k)+\frac{1}{\rho_{13}} T_{s} \bar{g}_{1} z_{1}^{2}(k+1) \\
& +c_{1} \rho_{12} z_{1}^{2}(k+1)+\frac{c_{1}}{\rho_{12}} z_{1}^{2}(k)+\rho_{11} \varepsilon_{1 M}^{2} \\
& +\frac{1}{\rho_{11}} z_{1}^{2}(k+1)-\frac{\delta_{1}}{\lambda_{1}} \widetilde{\omega}_{1}^{T}(k) \widetilde{\omega}_{1}(k) \\
& -\frac{\delta_{1}}{\lambda_{1}}\left\|\widehat{\omega}_{1}(k)\right\|^{2}+\frac{\delta_{1}}{\lambda_{1}}\left\|\omega_{1}^{*}\right\|^{2} \\
& +\delta_{1} \rho_{14}\left\|\widehat{\omega}_{1}(k)\right\|^{2}+\frac{\delta_{1} N_{1}}{\rho_{14}} z_{1}^{2}(k+1) .
\end{aligned}
$$

Finally, we obtain

$$
\begin{aligned}
\Delta L_{1}(k) \leq & -\gamma_{11} z_{1}^{2}(k+1)-\gamma_{12} z_{1}^{2}(k)-\gamma_{13}\left\|\widehat{\omega}_{1}(k)\right\|^{2} \\
& +\gamma_{14} z_{2}^{2}(k)+\gamma_{15},
\end{aligned}
$$

where $\gamma_{11}=1-\lambda_{1} N_{1}-\left(1 / \rho_{13}\right) T_{s} \bar{g}_{1}-k_{1} \rho_{12}-1 / \rho_{11}-\delta_{1} N_{1} / \rho_{14}$, $\gamma_{12}=1-c_{1} / \rho_{12}, \gamma_{13}=\delta_{1} / \lambda_{1}-\delta_{1}^{2} / \lambda_{1}-\delta_{1} \rho_{14}, \gamma_{14}=T_{s} \bar{g}_{1} \rho_{13}$, and $\gamma_{15}=\rho_{11} \varepsilon_{1 M}^{2}+\left(\delta_{1} / \lambda_{1}\right)\left\|\omega_{1}^{*}\right\|^{2}$.

(2) Define $z_{2}(k)=x_{2}(k)-x_{2 d}(k), X_{2}(k)=[V(k), h(k)$, $\left.x_{1}(k), x_{2}(k), x_{3}(k), x_{1 d}(k)\right]^{T}$. Since $f_{2}=0$ and $g_{2}=1$, we know

$$
z_{2}(k+1)=x_{2}(k)+T_{s} x_{3}(k)-x_{2 d}(k+1) .
$$

The uncertainty is defined and approximated with the NN:

$$
\begin{aligned}
U_{2}(k) & =x_{2 d}(k+1)-x_{2}(k) \\
& =\omega_{2}^{* T} \theta_{2}\left(X_{2}(k)\right)+\varepsilon_{2}\left(X_{2}(k)\right),
\end{aligned}
$$

where $\omega_{2}^{*}$ is the optimal NN weight vector to approximate $U_{2}(k)$. Denoting $\theta_{2}(k)=\theta_{2}\left(X_{2}(k)\right), \varepsilon_{2}(k)=\varepsilon_{2}\left(X_{2}(k)\right)$ is the NN construction error with upper bound $\varepsilon_{2 M}$.

Design virtual control $x_{3 d}(k)$ as

$$
x_{3 d}(k)=\frac{c_{2} z_{2}(k)+\widehat{\omega}_{2}^{T}(k) \theta_{2}(k)}{T_{s}},
$$

where $\widehat{\omega}_{2}$ is the estimation of $\omega_{2}^{*}$ and $c_{2}$ is the design constant.

Define $z_{3}(k)=x_{3}(k)-x_{3 d}(k)$. From (33) and (35), we have

$$
\begin{aligned}
z_{2}(k+1)= & c_{2} z_{2}(k)+T_{s} z_{3}(k)+\widetilde{\omega}_{2}^{T}(k) \theta_{2}(k) \\
& -\varepsilon_{2}(k)
\end{aligned}
$$

where $\widetilde{\omega}_{2}=\widehat{\omega}_{2}-\omega_{2}^{*}$.

The NN updating law is given as

$$
\widehat{\omega}_{2}(k+1)=\widehat{\omega}_{2}(k)-\lambda_{2} z_{2}(k+1) \theta_{2}(k)-\delta_{2} \widehat{\omega}_{2}(k) .
$$

Define the Lyapunov function candidate:

$$
L_{2}(k)=z_{2}^{2}(k)+\frac{\widetilde{\omega}_{2}^{T}(k) \widetilde{\omega}_{2}(k)}{\lambda_{2}} .
$$

Then,

$$
\begin{aligned}
\Delta L_{2}(k) \leq & -\gamma_{21} z_{2}^{2}(k+1)-\gamma_{22} z_{2}^{2}(k)-\gamma_{23}\left\|\widehat{\omega}_{2}(k)\right\|^{2} \\
& +\gamma_{24} z_{3}^{2}(k)+\gamma_{25},
\end{aligned}
$$

where $\gamma_{21}=1-\lambda_{2} N_{2}-\left(1 / \rho_{23}\right) T_{s}-c_{2} \rho_{22}-1 / \rho_{21}-\delta_{2} N_{2} / \rho_{24}$, $\gamma_{22}=1 / T_{s}-c_{2} / \rho_{22}, \gamma_{23}=\delta_{2} / \lambda_{2}-\delta_{2}^{2} / \lambda_{2}-\delta_{2} \rho_{24}, \gamma_{24}=\rho_{23} T_{s}$, and $\gamma_{25}=\rho_{21} \varepsilon_{2 M}^{2}+\left(\delta_{2} / \lambda_{2}\right)\left\|\omega_{2}^{*}\right\|^{2}$.

(3) According to the definition of $z_{3}(k)$, we have

$$
\begin{aligned}
z_{3}(k+1)= & x_{3}(k)+T_{s}\left[f_{3}(k)+g_{3}(k) u_{A}(k)\right] \\
& -x_{3 d}(k+1) .
\end{aligned}
$$

Define $X_{3}(k)=\left[V(k), h(k), x_{1}(k), x_{2}(k), x_{3}(k), x_{1 d}(k)\right]^{T}$. The uncertainty is defined as

$$
\begin{aligned}
U_{3}(k) & =-T_{s} f_{3}(k)-x_{3}(k)+x_{3 d}(k+1) \\
& =\omega_{3}^{* T} \theta_{3}(k)+\varepsilon_{3}(k),
\end{aligned}
$$


where $\omega_{3}^{*}$ is the optimal NN weight vector to approximate $U_{3}(k)$. Denoting $\theta_{3}(k)=\theta_{3}\left(X_{3}(k)\right), \varepsilon_{3}(k)=\varepsilon_{3}\left(X_{3}(k)\right)$ is the $\mathrm{NN}$ construction error with the upper bound $\varepsilon_{3 M}$.

The elevator deflection $u_{A}(k)$ is designed as

$$
u_{A}(k)=\frac{c_{3} z_{3}(k)+\widehat{\omega}_{3}^{T}(k) \theta_{3}(k)}{T_{s} g_{3}(k)},
$$

where $\widehat{\omega}_{3}$ is the estimation of $\omega_{3}^{*}$ and $c_{3}$ is a positive design constant. is

Combining (40) and (42), the error dynamics of $z_{3}(k+1)$

$$
z_{3}(k+1)=c_{3} z_{3}(k)+\widetilde{\omega}_{3}^{T}(k) \theta_{3}(k)-\varepsilon_{3}(k),
$$

where $\widetilde{\omega}_{3}=\widehat{\omega}_{3}-\omega_{3}^{*}$.

The NN updating law is given as

$$
\widehat{\omega}_{3}(k+1)=\widehat{\omega}_{3}(k)-\lambda_{3} z_{3}(k+1) \theta_{3}(k)-\delta_{3} \widehat{\omega}_{3}(k) .
$$

Define the Lyapunov function candidate:

$$
L_{3}(k)=z_{3}^{2}(k)+\frac{\widetilde{\omega}_{3}^{T}(k) \widetilde{\omega}_{3}(k)}{\lambda_{3}} .
$$

Then,

$$
\begin{aligned}
\Delta L_{3}(k) \leq & -\gamma_{31} z_{3}^{2}(k+1)-\gamma_{32} z_{3}^{2}(k)-\gamma_{33}\left\|\widehat{\omega}_{3}(k)\right\|^{2} \\
& +\gamma_{35},
\end{aligned}
$$

where $\gamma_{31}=1-\lambda_{3} N_{3}-c_{3} \rho_{32}-1 / \rho_{31}-\delta_{3} N_{3} / \rho_{34}, \gamma_{32}=1-c_{3} / \rho_{32}$, $\gamma_{33}=\delta_{3} / \lambda_{3}-\delta_{3}^{2} / \lambda_{3}-\delta_{3} \rho_{34}$, and $\gamma_{35}=\rho_{31} \varepsilon_{3 M}^{2}+\left(\delta_{3} / \lambda_{3}\right)\left\|\omega_{3}^{*}\right\|^{2}$.

By selecting $\rho_{3 i}, i=1,2,3,5$, and $k_{3}, \lambda_{3}, \delta_{3}$, we know that $\gamma_{3 i}>0$ can be guaranteed. From (46), if $\left|z_{3}(k)\right|>\sqrt{\gamma_{35} / \gamma_{32}}$, then $\Delta L_{3} \leq 0$. So $z_{3}(k)$ is bounded. This further implies that $z_{2}(k)$ is bounded from (39). Then, from (32), $z_{1}(k)$ is bounded.

Now, the following theorem can be obtained.

Theorem 4. Consider system (18) with controllers (22), (35), and (42) and NN updating laws (24), (38), and (44). All the signals involved are bounded.

\section{Simulation}

For the velocity subsystem, the controller in [42] is simply employed. In this section, we verify the effectiveness and performance of the proposed guidance law and adaptive neural controller. The hypersonic missile starts from the state at $h=118110 \mathrm{ft}, V=15060 \mathrm{ft} / \mathrm{s}, \alpha=0 \mathrm{deg}, \gamma=0 \mathrm{deg}$, and $q=0 \mathrm{deg} / \mathrm{s}$. The target starts from the state at $h=119090 \mathrm{ft}$ and $V=2230 \mathrm{ft} / \mathrm{s}$ and has an upward maneuvering overload $n=1.5 \mathrm{~g}$ in the interception phase.

The parameters for the guidance law are selected as $k=$ 2.5, $\varepsilon=0.1$, and $\delta=0.5$. The parameters for the controller are selected as $T_{s}=0.005 s, c_{1}=0.9, c_{2}=0.95, c_{3}=0.95$, $c_{V}=0.95, \lambda_{i}=0.002, \delta_{i}=0.05, i=1,2,3, \lambda_{v}=0.02$, and $\varepsilon_{0}=0.0001$. The number of NN nodes is 50 . The initial NN

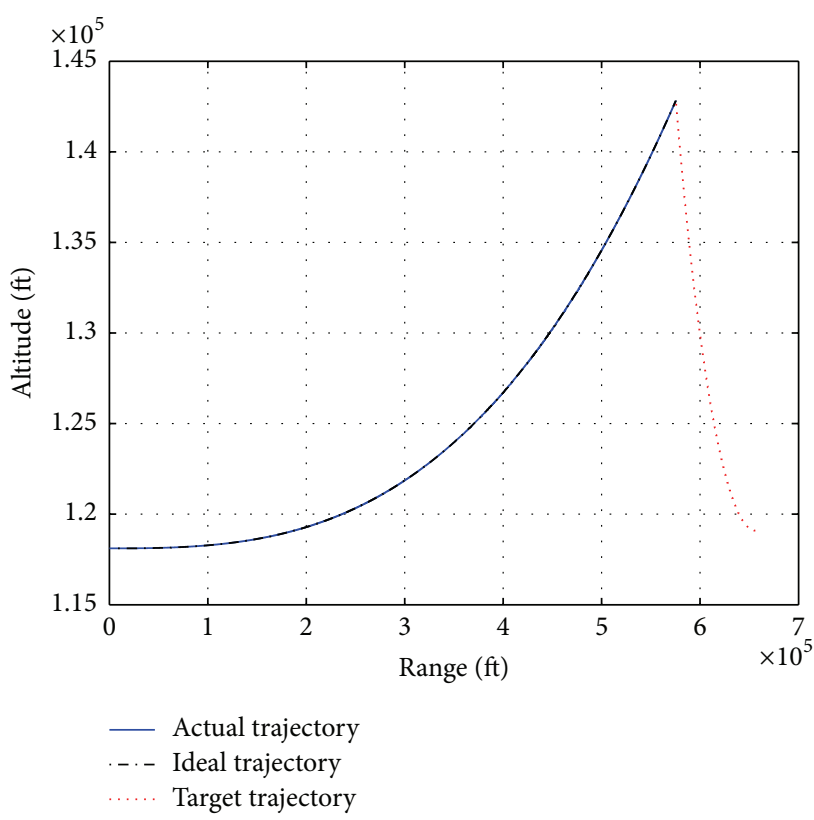

FIGURE 3: Interception trajectory.

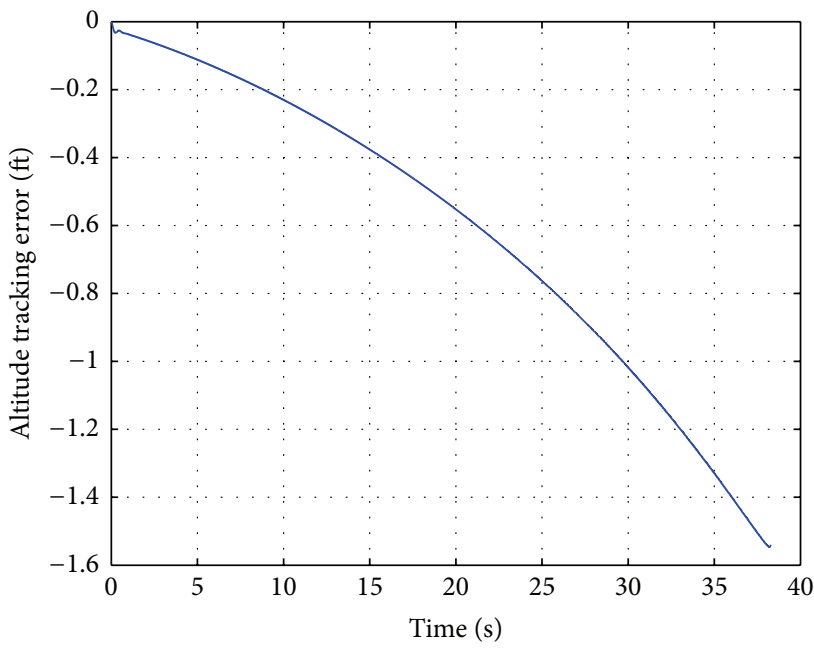

FIGURE 4: Altitude tracking error.

weights for $\widehat{g}_{v}$ are set as 5, while for other NNs the weights are set as zero.

The simulation results are shown in Figures 3-12. From the target interception in Figures 3 and 4, it can be observed that the target (dotted line) maneuvering upward rapidly is impacted by the hypersonic missile (solid line) which tracks the trajectory (dash dot line) calculated precisely by the sliding mode guidance. From the flight path angle tracking in Figures 5 and 6, it can be concluded that the altitude control law tracks the flight path angle at the desired value calculated accurately by the guidance law. From the velocity tracking in Figures 7 through 10, it can be concluded that the velocity controller achieves good performance of keeping the velocity at constant value. The throttle setting changes with the value of angle of attack, indicating the coupling of aerodynamics 

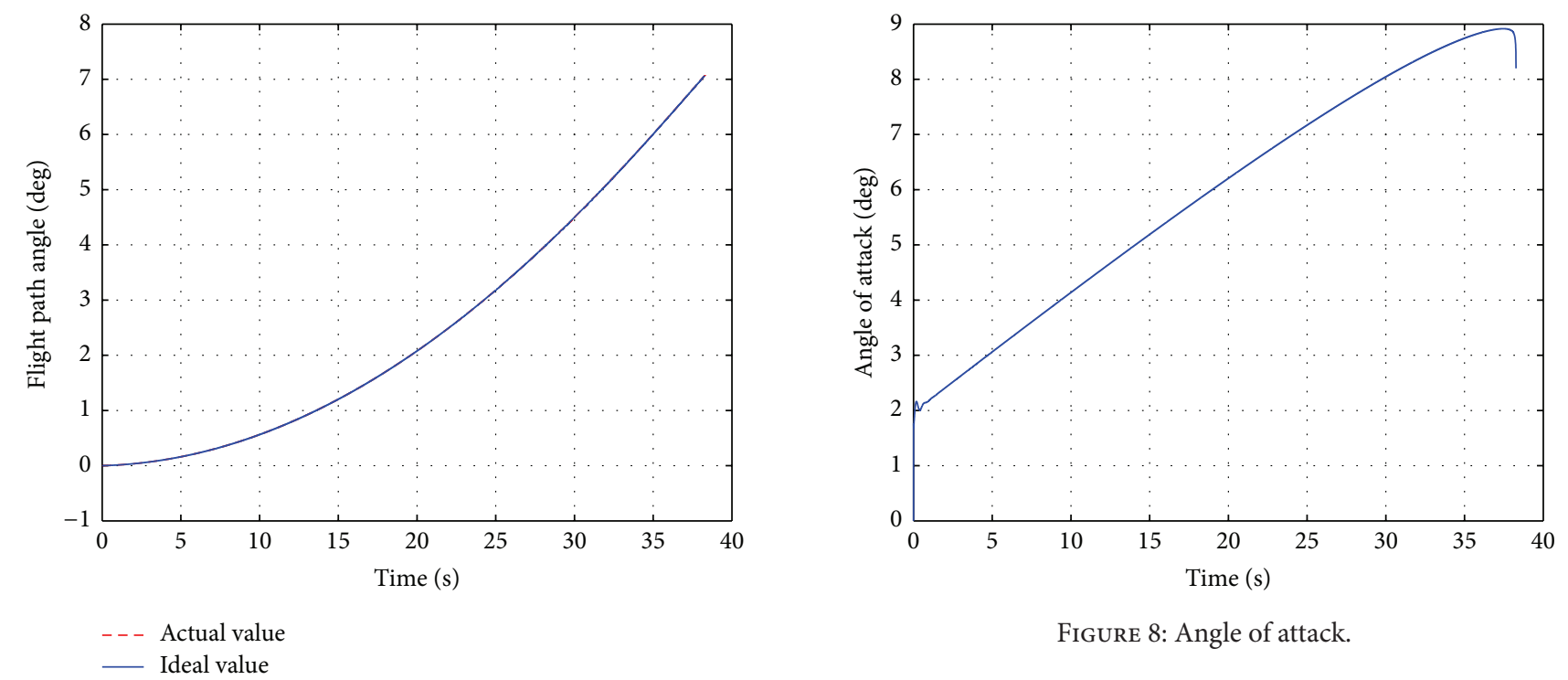

Figure 8: Angle of attack.

FIGURE 5: Flight path angle.

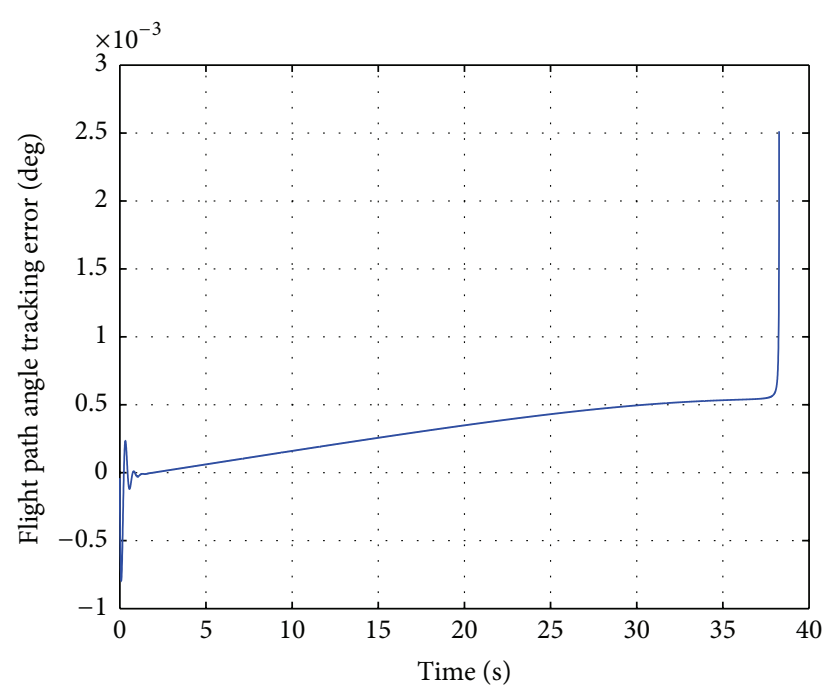

FIgURE 6: Flight path angle tracking error.

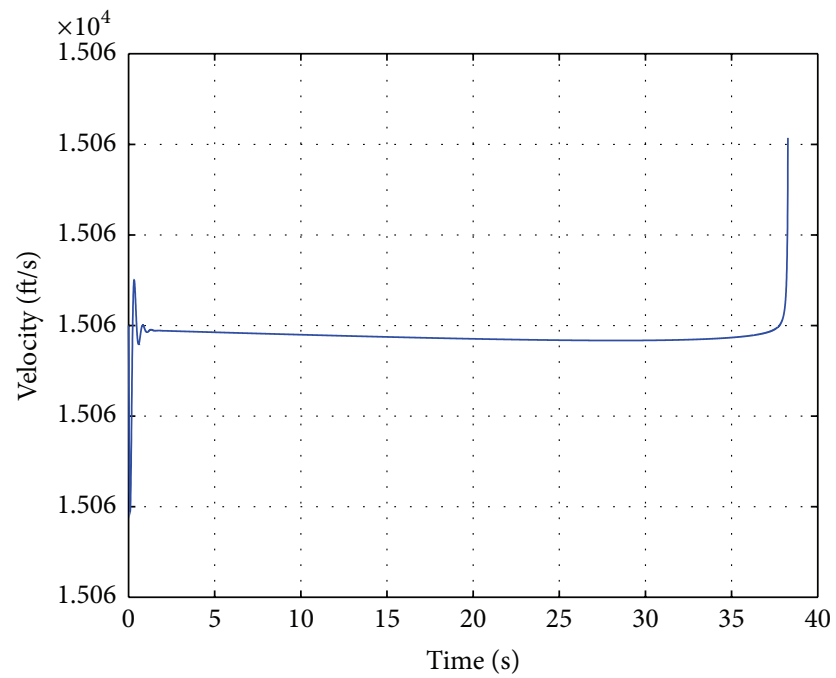

Figure 7: Velocity.

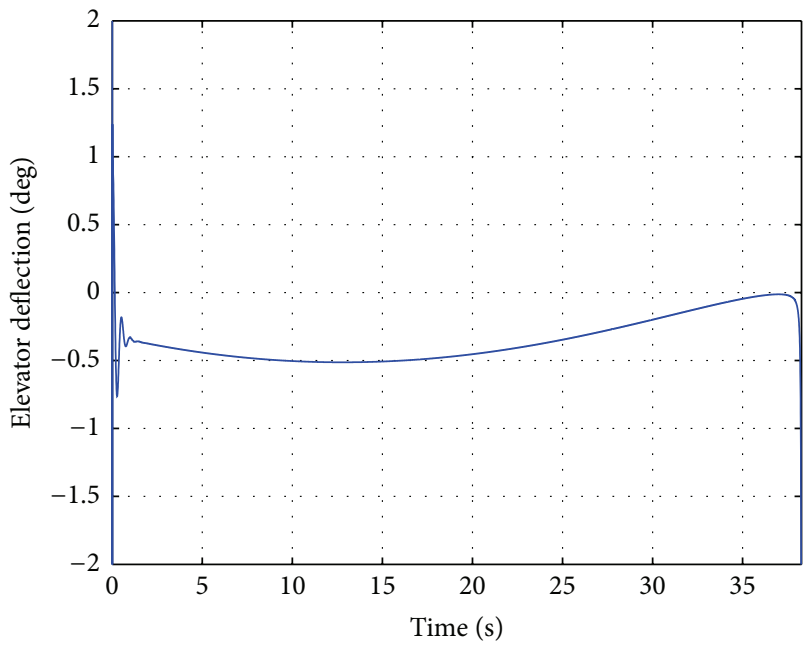

FIGURE 9: Elevator deflection.

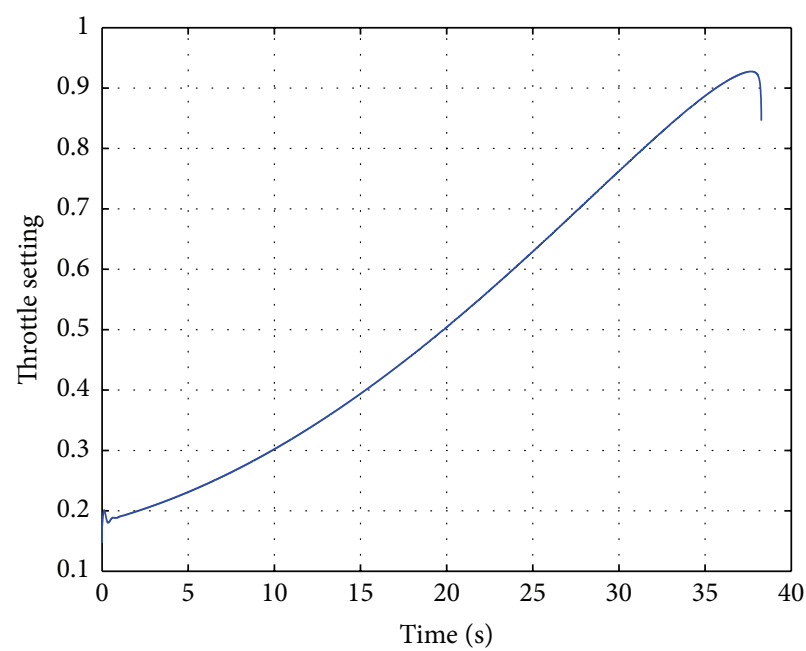

FIGURE 10: Throttle setting. 


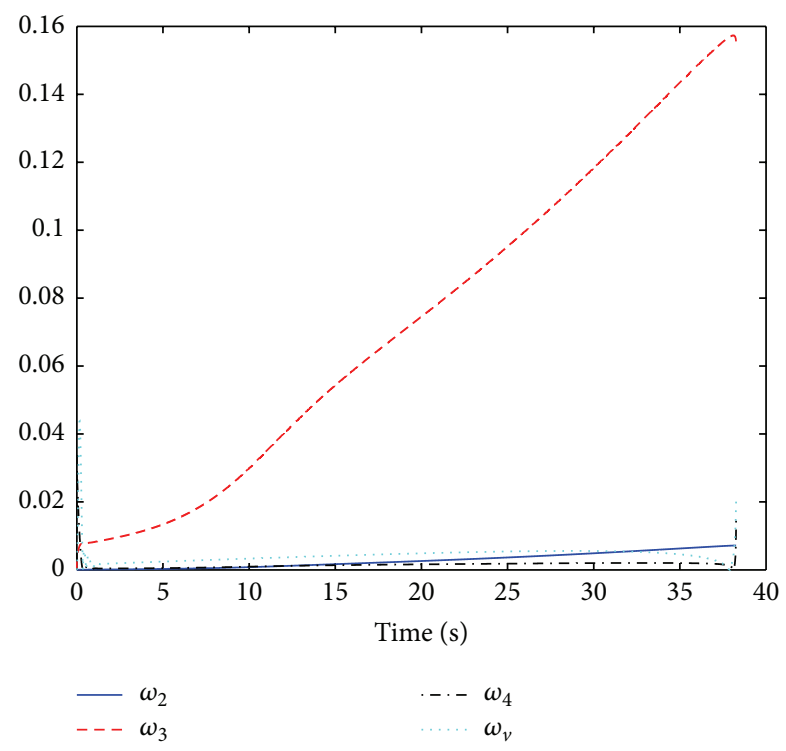

FIGURE 11: NN weights response (1).

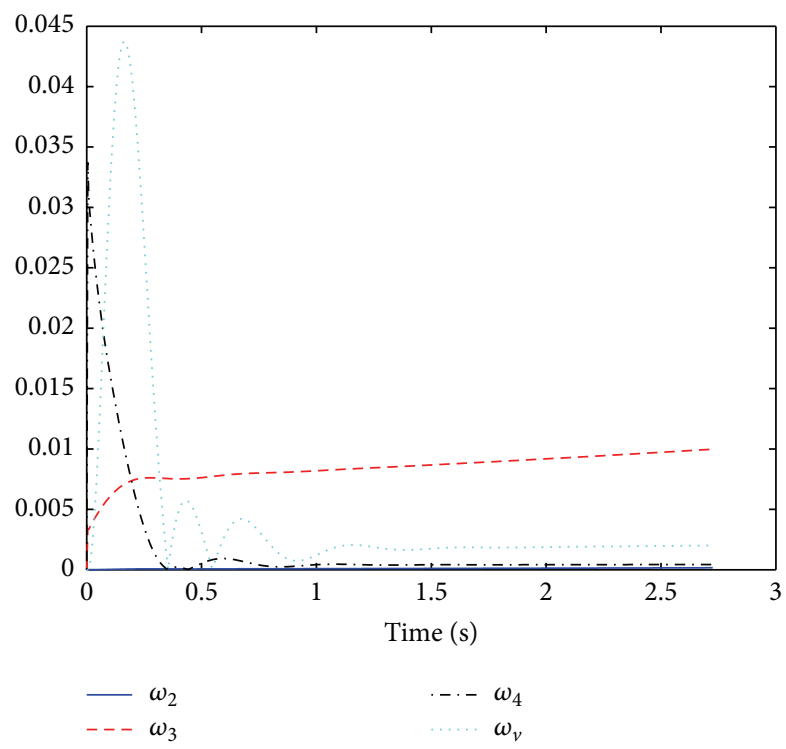

FIGURE 12: NN weights response (2).

and propulsion. Figures 7 through 10 depict the changes in the velocity, angle of attack, and control input which oscillate in the first second and then become stable. The reason for oscillation can be found in the tracking of the NN weights depicted in Figures 11 and 12. The NN has learned the dynamic characteristics of the hypersonic missile.

\section{Conclusion and Future Work}

Taking the target maneuver into consideration, this paper proposes the sliding mode guidance law and adaptive neural controller for the hypersonic missile via the back-stepping method. The flight path angle is designed as the guidance command instead of normal acceleration. The nominal part of the nonlinearity during each step is eliminated and the NN is used to approximate the system uncertainty. Simulation results show the effectiveness of the proposed guidance and control laws. For future work, the energy management control should be considered to derive more effective velocity control.

In this paper, only one-step-ahead model is used for controller design. For future work, we will work on how to develop a new algorithm based on the equivalent prediction model developed in [43]. Also, the fault tolerant control [44] is of interest since it can guarantee flight safety. In case of time-varying disturbance, the disturbance observer based control [45] can be applied.

\section{Competing Interests}

The authors declare that they have no competing interests.

\section{Acknowledgments}

This work was supported by the National Natural Science Foundation of China under Grants 61104195, 90816027, and 91216104 and by the NWPU Basic Research Fund under Grant GEKY1003.

\section{References}

[1] B. Xu and Z. Shi, "An overview on flight dynamics and control approaches for hypersonic vehicles," Science China Information Sciences, vol. 58, no. 7, Article ID 070201, 19 pages, 2015.

[2] H. J. Xu, M. D. Mirmirani, and P. A. Ioannou, "Adaptive sliding mode control design for a hypersonic flight vehicle," Journal of Guidance, Control, and Dynamics, vol. 27, no. 5, pp. 829-838, 2004.

[3] B. Xu, X. Huang, D. Wang, and F. Sun, "Dynamic surface control of constrained hypersonic flight models with parameter estimation and actuator compensation," Asian Journal of Control, vol. 16, no. 1, pp. 162-174, 2014.

[4] B. Xu, "Robust adaptive neural control of flexible hypersonic flight vehicle with dead-zone input nonlinearity," Nonlinear Dynamics, vol. 80, no. 3, pp. 1509-1520, 2015.

[5] Z. D. Wilcox, W. MacKunis, S. Bhat, R. Lind, and W. E. Dixon, "Robust nonlinear control of a hyper-sonic aircraft in the presence of aerothermoelastic effects," in Proceedings of the American Control Conference (ACC '09), pp. 2533-2538, IEEE, St. Louis, Mo, USA, June 2009.

[6] H. Buschek and A. J. Calise, "Uncertainty modeling and fixedorder controller design for a hypersonic vehicle model," Journal of Guidance, Control, and Dynamics, vol. 20, no. 1, pp. 42-48, 1997.

[7] M. Zerar, F. Cazaurang, and A. Zolghadri, "Coupled linear parameter varying and flatness-based approach for space reentry vehicles guidance," IET Control Theory and Applications, vol. 3, no. 8, pp. 1081-1092, 2009.

[8] Q. Wang and R. F. Stengel, "Robust nonlinear control of a hypersonic aircraft," Journal of Guidance, Control, and Dynamics, vol. 23, no. 4, pp. 577-585, 2000.

[9] C. I. Marrison and R. F. Stengel, "Design of robust control systems for a hypersonic aircraft," Journal of Guidance, Control, and Dynamics, vol. 21, no. 1, pp. 58-63, 1998. 
[10] S. Mehta, W. MacKunis, S. Subramanian, and C. Pasiliao, "Nonlinear control of hypersonic missiles for maximum target penetration," in Proceedings of the AIAA Guidance, Navigation, and Control Conference, Guidance, Navigation, and Control and Co-located Conferences, Minneapolis, Minn, USA, August 2012.

[11] P. Yu, Y. B. Shtessel, and C. Edwards, "Adaptive continuous higher order sliding mode control of air breathing hypersonic missile for maximum target penetration," in Proceedings of the AIAA Guidance, Navigation, and Control Conference, AIAA SciTech. American Institute of Aeronautics and Astronautics, Kissimmee, Florida, 2015.

[12] V. I. Utkin, Sliding Modes in Control and Optimization, Springer, New York, NY, USA, 1992.

[13] J.-J. E. Slotine and W. Li, Applied Nonlinear Control, vol. 199, Prentice-Hall, Englewood Cliffs, NJ, USA, 1991.

[14] K. R. Babu, I. G. Sarma, and K. N. Swamy, "Switched bias proportional navigation for homing guidance against highly maneuvering targets," Journal of Guidance, Control, and Dynamics, vol. 17, no. 6, pp. 1357-1363, 1994.

[15] J. Moon, K. Kim, and Y. Kim, "Design of missile guidance law via variable structure control," Journal of Guidance, Control, and Dynamics, vol. 24, no. 4, pp. 659-664, 2001.

[16] D. Zhou, C. Mu, and W. Xu, "Adaptive sliding-mode guidance of a homing missile," Journal of Guidance, Control, and Dynamics, vol. 22, no. 4, pp. 589-594, 1999.

[17] Y. B. Shtessel and C. H. Tournes, "Integrated higher-order sliding mode guidance and autopilot for dual-control missiles," Journal of Guidance, Control, and Dynamics, vol. 32, no. 1, pp. 79-94, 2009.

[18] T. Shima, M. Idan, and O. M. Golan, "Sliding-mode control for integrated missile autopilot guidance," Journal of Guidance, Control, and Dynamics, vol. 29, no. 2, pp. 250-260, 2006.

[19] Y. B. Shtessel, I. A. Shkolnikov, and A. Levant, "Smooth secondorder sliding modes: missile guidance application," Automatica, vol. 43, no. 8, pp. 1470-1476, 2007.

[20] P. V. Kokotovic, “The joy of feedback: nonlinear and adaptive: 1991 bode prize lecture," IEEE Control Systems, vol. 12, no. 3, pp. 7-17, 1992.

[21] M. Chen, S. S. Ge, and B. V. E. How, "Robust adaptive neural network control for a class of uncertain MIMO nonlinear systems with input nonlinearities," IEEE Transactions on Neural Networks, vol. 21, no. 5, pp. 796-812, 2010.

[22] L. J. Chen and K. S. Narendra, "Nonlinear adaptive control using neural networks and multiple models," Automatica, vol. 37, no. 8, pp. 1245-1255, 2001.

[23] L. Fiorentini, A. Serrani, M. A. Bolender, and D. B. Doman, "Nonlinear robust adaptive control of flexible air-breathing hypersonic vehicles," Journal of Guidance, Control, and Dynamics, vol. 32, no. 2, pp. 402-417, 2009.

[24] B. Xu, Z. Shi, C. Yang, and F. Sun, "Composite neural dynamic surface control of a class of uncertain nonlinear systems in strict-feedback form," IEEE Transactions on Cybernetics, vol. 44, no. 12, pp. 2626-2634, 2014.

[25] Z.-G. Wu, P. Shi, H. Su, and J. Chu, "Local synchronization of chaotic neural networks with sampled-data and saturating actuators," IEEE Transactions on Cybernetics, vol. 44, no. 12, pp. 2635-2645, 2014.

[26] B. Xu, Q. Zhang, and Y. Pan, "Neural network based dynamic surface control of hypersonic flight dynamics using small-gain theorem," Neurocomputing, vol. 173, part 3, pp. 690-699, 2016.
[27] M. Chen, P. Shi, and C. C. Lim, "Adaptive neural fault-tolerant control of a 3-dof model helicopter system," IEEE Transactions on Systems, Man, and Cybernetics: Systems, vol. 46, no. 2, pp. 260-270, 2015.

[28] C. Yang, Z. Li, R. Cui, and B. Xu, "Neural network-based motion control of an underactuated wheeled inverted pendulum model," IEEE Transactions on Neural Networks and Learning Systems, vol. 25, no. 11, pp. 2004-2016, 2014.

[29] B. Xu, Z. Shi, and C. Yang, "Composite fuzzy control of a class of uncertain nonlinear systems with disturbance observer," Nonlinear Dynamics, vol. 80, no. 1-2, pp. 341-351, 2015.

[30] Z. Wu, P. Shi, H. Su, and R. Lu, "Dissipativity-based sampleddata fuzzy control design and its application to truck-trailer system," IEEE Transactions on Fuzzy Systems, vol. 23, no. 5, pp. 1669-1679, 2015.

[31] B. Xu, C. Yang, and Y. Pan, "Global neural dynamic surface tracking control of strict-feedback systems with application to hypersonic flight vehicle," IEEE Transactions on Neural Networks and Learning Systems, vol. 26, no. 10, pp. 2563-2575, 2015.

[32] Y.-J. Liu, L. Tang, S. Tong, and C. L. P. Chen, "Adaptive NN controller design for a class of nonlinear MIMO discrete-time systems," IEEE Transactions on Neural Networks and Learning Systems, vol. 26, no. 5, pp. 1007-1018, 2015.

[33] Y.-J. Liu, L. Tang, S. Tong, C. L. P. Chen, and D.-J. Li, "Reinforcement learning design-based adaptive tracking control with less learning parameters for nonlinear discrete-time MIMO systems," IEEE Transactions on Neural Networks and Learning Systems, vol. 26, no. 1, pp. 165-176, 2015.

[34] B. Xu, C. Yang, and Z. Shi, "Reinforcement learning output feedback NN control using deterministic learning technique," IEEE Transactions on Neural Networks and Learning Systems, vol. 25, no. 3, pp. 635-641, 2014.

[35] C. Yang, L. Zhai, S. G. Shuzhi, T. Chai, and H. L. Tong, "Adaptive model reference control of a class of MIMO discrete-time systems with compensation of nonparametric uncertainty," in Proceedings of the American Control Conference (ACC '08), pp. 4111-4116, IEEE, Seattle, Wash, USA, June 2008.

[36] S. Janardhanan and B. Bandyopadhyay, "Multirate output feedback based robust quasi-sliding mode control of discrete-time systems," IEEETransactions on Automatic Control, vol. 52, no. 3, pp. 499-503, 2007.

[37] A. Chaudhuri and M. S. Bhat, "Output feedback-based discretetime sliding-mode controller design for model aircraft," Journal of Guidance, Control, and Dynamics, vol. 28, no. 1, pp. 177-181, 2005.

[38] N. K. Lincoln and S. M. Veres, "Application of discrete time sliding mode control to a spacecraft in $6 \mathrm{DoF}$ with parameter identification," International Journal of Control, vol. 83, no. 11, pp. 2217-2231, 2010.

[39] I. M. Mareels, H. B. Penfold, and R. J. Evans, "Controlling nonlinear time-varying systems via Euler approximations," Automatica, vol. 28, no. 4, pp. 681-696, 1992.

[40] B. Xu, F. C. Sun, C. G. Yang, D. X. Gao, and J. X. Ren, "Adaptive discrete-time controller design with neural network for hypersonic flight vehicle via back-stepping," International Journal of Control, vol. 84, no. 9, pp. 1543-1552, 2011.

[41] M. A. Bolender and D. B. Doman, "Nonlinear longitudinal dynamical model of an air-breathing hypersonic vehicle," Journal of Spacecraft and Rockets, vol. 44, no. 2, pp. 374-387, 2007.

[42] B. Xu, Y. Fan, and S. Zhang, "Minimal-learning-parameter technique based adaptive neural control of hypersonic flight 
dynamics without back-stepping," Neurocomputing, vol. 164, pp. 201-209, 2015.

[43] B. Xu and Y. Zhang, "Neural discrete back-stepping control of hypersonic flight vehicle with equivalent prediction model," Neurocomputing, vol. 154, pp. 337-346, 2015.

[44] B. Xu, Y. Guo, Y. Yuan, Y. Fan, and D. Wang, "Fault-tolerant control using command-filtered adaptive back-stepping technique: application to hypersonic longitudinal flight dynamics," International Journal of Adaptive Control and Signal Processing, 2015.

[45] M. Chen and S. S. Ge, "Adaptive neural output feedback control of uncertain nonlinear systems with unknown hysteresis using disturbance observer," IEEE Transactions on Industrial Electronics, vol. 62, no. 12, pp. 7706-7716, 2015. 


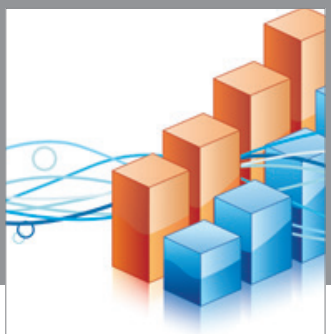

Advances in

Operations Research

vatem alat4

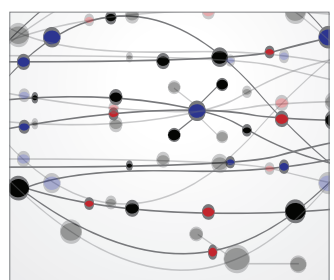

\section{The Scientific} World Journal
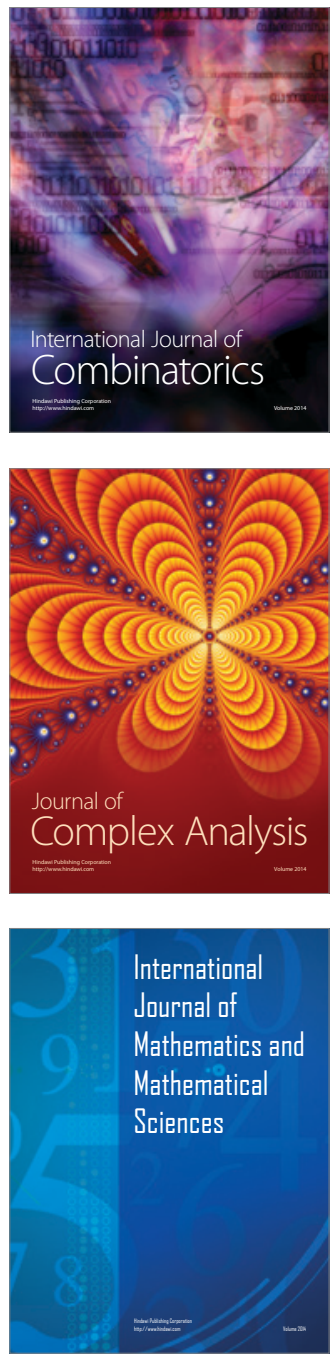
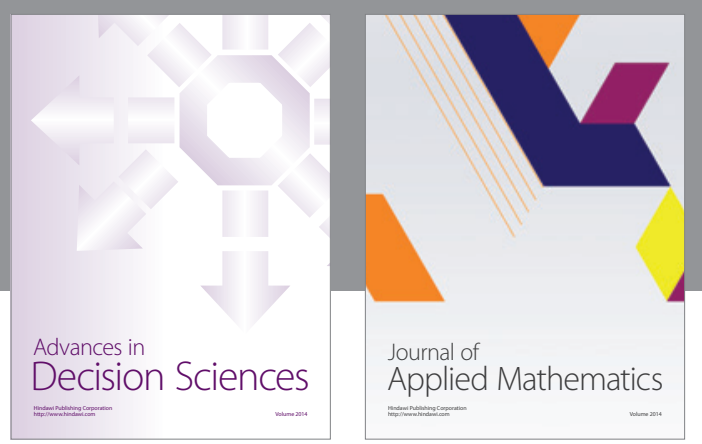

Algebra

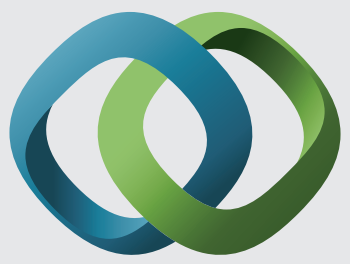

\section{Hindawi}

Submit your manuscripts at

http://www.hindawi.com
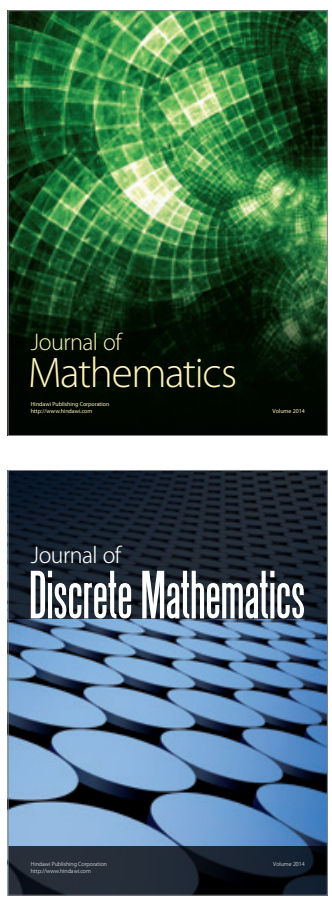

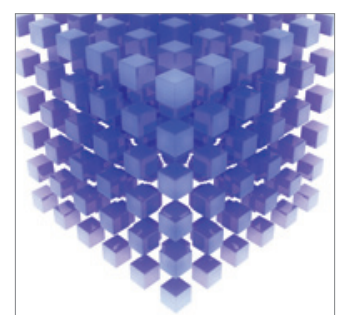

Mathematical Problems in Engineering
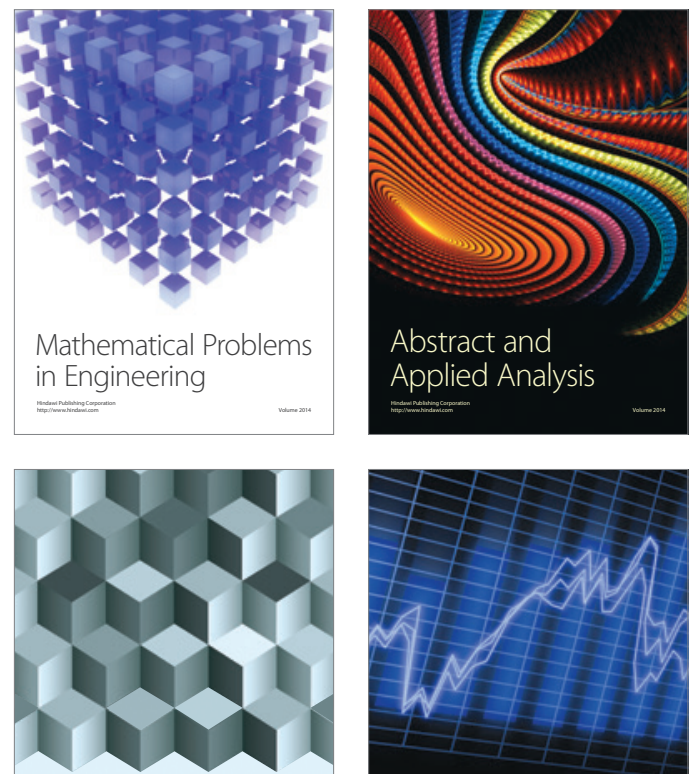

Journal of

Function Spaces

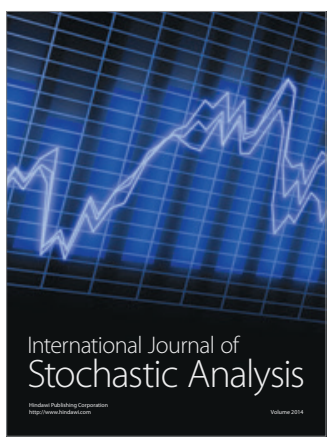

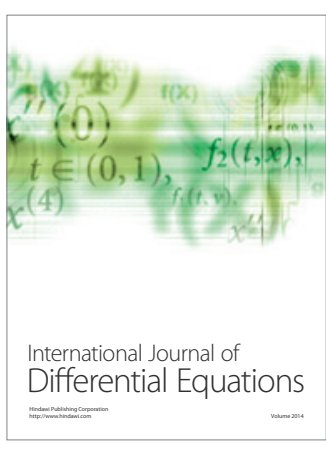
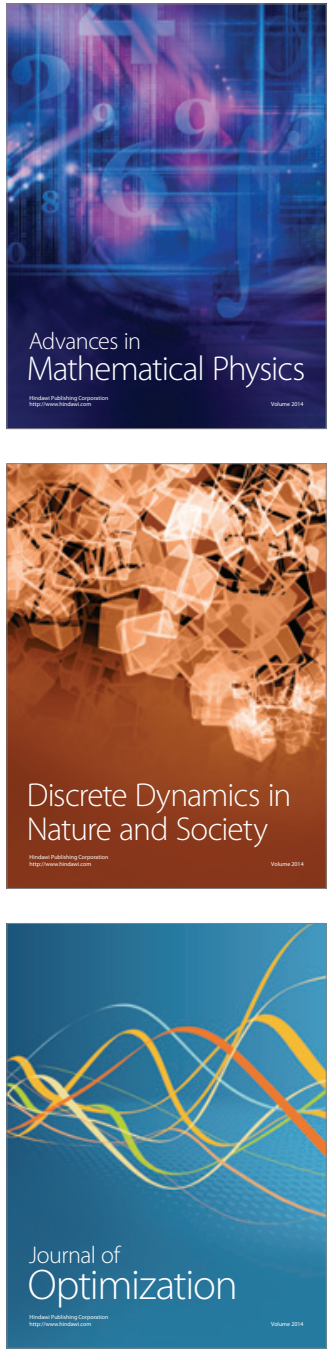